FOLIA SCANDINAVICA VOL. 24 POZNAŃ 2018 DOI: $10.2478 /$ fsp-2018-0003
S sciendo

PRESSto.

\title{
DER FREMDE IM KÖNIGREICH DER DÄNEN. DAS WORT, DER DISKURS UND DIE FREMDHEIT IN BRONISŁAW ŚWIDERSKIS ROMAN SEOWA OBCEGO
}

\author{
PIOTR DE BOŃCZA BUKOWSKI \\ Jagiellonian University in Kraków
}

ABSTRACT. The prose work of the Polish exile writer Bronisław Świderski, who has been living in Denmark since 1970, explores strangeness - an important topic of modern literature. Świderski addresses strangeness not only as an individual experience, but also as a social problem. In this article, I would like to take a closer look at the analysis of foreignness and the psyche of an immigrant in Świderski's award-winning novel Stowa obcego (1998). From many problems addressed in the novel, which are directly or indirectly connected with strangeness, I want to take out one aspect. At this point I will be interested in the relationship between language (discourse) and strangeness.

\section{1. ŚWIDERSKIS ANALYTIK DER FREMDHEIT}

Das literarische Schaffen mancher Schriftsteller zeichnet sich durch einen oft schwer zu erfassenden Themenreichtum aus, das Werk anderer Autoren hingegen besticht durch die Intensität, mit welcher es sich mit einem einzigen Thema auseinandersetzt. Das Prosawerk des seit 1970 in Dänemark lebenden polnischen Exilschriftstellers Bronisław Świderski umkreist und erforscht das große Thema der Fremdheit. Świderski thematisiert Fremdheit nicht nur als individuelle Erfahrung (welche er mit seiner eigenen Biographie - eines polnischen Immigranten mit jüdischen Wurzeln - in Zusammenhang bringt), sondern auch als gesellschaftliches Problem. In seinen Romanen erteilt er das Wort einem Immigranten, der sein eigenes Bewusstsein analysiert, seine Sprache erforscht, über seine Stellung in der neuen AsylHeimat - dem Königreich Dänemark - kritisch reflektiert. Świderskis Erzählerfiguren, 
oft als seine Alter Egos konstruiert, sprechen gleichzeitig für sich selbst und für alle Immigranten, für alle Fremden dieser Erde. Die individuelle, historisch bestimmbare Erfahrung der Fremdheit wird im Werk dieses Autors zu einer allgemeinen Theorie der Fremdheit kondensiert, die kommunikationstheoretisch und philosophisch unterbaut ist.

Bausteine für eine Analytik der Fremdheit und damit auch für die Studie der Psyche des Immigranten sind eigentlich in allen Werken von Bronisław Świderski zu finden - also sowohl in diskursiven Texten (vor allem Søren Kierkegaard gewidmet ${ }^{1}$ ), als auch in fiktionalisierten Autobiographien (wie Asystent śmierci, dt. Der Assistent des Todes) und autobiographisch fundierten Fiktionen (wie Stowa obcego). ${ }^{2}$ In dem vorliegenden Artikel möchte ich diese Analytik im preisgekrönten Roman Stowa obcego (dt. Die Worte des Fremden) aus dem Jahre 1998 näher betrachten. ${ }^{3}$ Aus der Fülle der mit Fremdheit direkt oder indirekt verbundenen und miteinander verflochtenen Probleme, die in diesem Werk thematisiert werden ${ }^{4}$, will ich einen Aspekt herausnehmen und fokussieren. Mich wird an dieser Stelle das Verhältnis von Sprache und Fremdheit interessieren.

Man kann behaupten, dass das Werk selbst diese Perspektive aufzwingt, da es schon in seinem Titel Sprache mit Fremdheit verknüpft, doch im Fall der vorliegenden Analyse ergab sie sich aus der Lektüre von Julia Kristevas Studie „Tokkata und Fuge für den Fremden" aus dem Buch Fremde sind wir uns selbst (franz. Etrangers à nous-mêmes, 1988). In einem Text, der tatsächlich an polyphone Musik denken lässt, versucht die bulgarisch-französische Literaturwissenschaftlerin und Schriftstellerin das facettenreiche Phänomen der Fremdheit zu beschreiben. Sie entwickelt eine mehrstimmige Darstellung, in welcher das Autobiographische (das Immigrantendasein, das Fremdsein in Frankreich), die existentielle, psychoanalytische und historische Analyse und schließlich auch die Literaturkritik zu Wort kommen.

${ }^{1}$ Siehe Świderski, 2015. Vgl. aber auch Świderski, 2012a: 111-113.

2 Zur Rolle der autobiographischen Konvention in Świderskis Schaffen vgl. Świderski, 2012b: 133-134.

3 Świderski, 1998; fortan: SO mit der Seitenangabe. Alle Zitate aus diesem Roman in meiner Übersetzung. Das Buch wurde in einem vom Krakauer Verlagshaus „Znak“ veranstalteten Roman-Wettbewerb mit dem 2. Preis ausgezeichnet .

${ }^{4}$ Der Inhalt des vielschichtigen Romans lässt sich nicht leicht zusammenfassen. Der Verleger („Znak“) wagt den Versuch: „Der Protagonist des Romans, der Pole B., will das Rätsel lösen: In welcher Sprache schreibt der Fremde, der in Dänemark politisches Asyl sucht? Von der Erklärung dieses Rätsels hängt seine Karriere ab. Doch vielleicht ist der geheimnisvolle Fremde ein Teil der Liebesstrategie der Kopenhagener Polizistin Lone, die bis über beide Ohren in B. verliebt ist? Auch Lone rätselt: Warum liebt B. sie nicht? Warum sind ihre Weiblichkeit und Leidenschaft nicht genug, um eine Beziehung zu schaffen? Warum braucht jedes Paar die Anwesenheit eines dritten, des Fremden?" (Stowa obcego, Bronisław Świderski, 2018; Übers. des Verfassers) 


\section{JULIA KRISTEVA UND DIE FIGUREN DES FREMDEN}

„Können wir die anderen (er)leben?“ - fragt Kristeva gleich am Anfang ihrer Studie (Kristeva, 1990:11). Angesichts der seit Jahren anwachsenden Zahl der Immigranten ist es in der Tat eine der dringlichsten Fragen - sowohl in Europa, als auch anderswo. Doch solange die Einheimischen nicht aufhören, auf ihrer individuellen Einheitlichkeit zu beharren und diese zu glorifizieren, solange sie ihre eigene Fremdheit (und damit die eigenen „Inkohärenzen“" und „Abgründe") verdrängten, sei kein Zusammenleben mit den Nicht-Einheimischen möglich (ibidem, 1990:12). Kristevas Devise heißt: „Die Fremdheit des Fremden nicht zu fixieren, zu verdinglichen suchen. Sie gerade nur berühren, sie streifen, ohne ihr eine endgültige Struktur zu geben“ (ibidem, 1990:12). Deshalb bewegt sich ihre Analyse von einer Figur der Fremdheit zur anderen, wobei sich die verschiedenen Themen und Motive miteinander verflechten (vgl. ibidem, 1990:13). Und so schreibt Kristeva über die „verborgene Verletzung“ des Exilierten, der „unverstanden von einer geliebten, aber unaufmerksamen, zurückhaltenden oder besorgten Mutter" ihr fremd sei und unter seiner Fremdheit leidet. (ibidem, 1990:15) Ein erschütterndes Bild des mutterlos gewordenen Fremden, des „Fanatikers der Absenz“, entdeckt Kristeva bei Albert Camus in dessen Roman L'Etranger (dt. Der Fremde), doch eine nicht weniger suggestive Erzählung von einem Fremden und seiner abwesenden geliebten-gehassten Mutter finden wir in Świderskis Asystent śmierci. Die Mutter des Ich-Erzählers („,des Autors und Erzählers“; Świderski, 2007:501) zeigte ihm offen ihre Abneigung: „Sie hat mich niemals geküsst, niemals umarmt", erinnert er sich (ibidem, 2007:117). Mehr noch: sie hat ihn mit ihrem eigenen Fremdheitsgefühl infiziert noch bevor er aus Polen emigrierte. „Sie sagte zu mir: denk immer daran, dass du anders bist (sie sagte nicht Jude, nur: anders!) und jederzeit getötet werden kannst“" (ibidem, 2007:140) - so offenbart sich die Genealogie von Bronisławs Andersheit und Fremdheit.

Auch weitere von Kristeva besprochene Figuren bzw. Motive, die mit dem Fremdsein zusammenhängen, können mit Świderskis Narrationen im Zusammenhang gebracht werden. So etwa die „Indifferenz“ des Fremden, seine (scheinbar) gefühllose und distanzierte Stellung gegenüber der umgebenden Welt, die von seiner Verletzbarkeit zeugt (Kristeva, 1990:17). Für Świderskis Erzähler-Ich ist die Indifferenz oft eine Zuflucht, in die er sich vor der Verzweiflung rettet. Die Existenz des heimatlosen Wahl-Dänen, der als Jude angeprangert sein Vaterland verlassen musste und nun abseits der Gesellschaft lebt, wird durch Kristevas Darstellung verallgemeinert und psychoanalytisch beleuchtet: „Keinem Ort zugehörig, keiner Zeit, keiner Liebe. Der Ursprung ist verloren, die Verwurzelung unmöglich, eine 
Erinnerung, die sich immer tiefer gräbt, eine Gegenwart mit offenem Horizont" (ibidem, 1990:17).

Ebenso der in dem Fremden „tief verwurzelte Narzissmus“, sein Maskenspiel, sein Balancieren zwischen Zerstückelung und Einheit, aber auch der Hass, welcher ihn umgibt und infiziert - all das ist für die existenzielle Lage des Fremden essenziell (ibidem, 1990:17-24). Es sollte uns also nicht wundern, dass diese Figuren nicht nur in Świderskis Werken, sondern auch in anderen Texten der Migranten auffindbar sind. Doch der - in meinen Augen wichtigste Zugang zu dem Migranten-Ich, welcher in die tiefste Erfahrungsschicht der Fremdheit führt, erfolgt durch das fremde Wort. In ihrer Studie betrachtet Julia Kristeva dieses Wort aus mehreren Perspektiven.

„Nicht seine Muttersprache sprechen“: was heißt es für den Fremden? fragt Kristeva (ibidem,1990:24). Die neue Sprache ist wie neue Haut, wie eine neue Identität. Aber die Worte klingen immer fremd, die Sprachfehler verraten den Fremden, der ja ,die Sprache eines anderen“ spricht (ibidem, 1990:25). "Zwischen zwei Sprachen geraten, ist euer Element somit das Schweigen“- so wendet sich Kristeva an die Fremden unserer Welt und eben diese Erfahrung bringt auch der um das Wort bangende Fremde in Świderskis Roman Stowa obcego mehrmals zum Ausdruck.

Die zweite Perspektive kann mit dem Postulat „nicht übereinstimmen“ zusammengefasst werden (ibidem, 1990:26). Es ist eine Ethik des Widerstandes gegen den herrschenden Diskurs der Einheimischen, welchen der Fremde zu dekonstruieren versucht. Denn er weiß, „dass die Worte, das Lächeln, der Zorn, die Urteile, der Geschmack des Einheimischen übertrieben, nicht fundiert oder einfach ungerecht und falsch sind, und dass er - stolz auf eigenem Boden zu stehen - sich nie fragt, ob man auch anders sagen, denken, tun könnte.“ (ibidem, 1990:26)

In Świderskis Schaffen kommt immer wieder die Einsicht zum Ausdruck, dass der entwurzelte Immigrant ein ethisches Recht, ja sogar eine Pflicht hat, den Standpunkt der Einheimischen zu relativieren; er fühlt sich dazu berechtigt, ein Wort an sie zu richten, das ihre Sicherheit und ihr Vertrauen in die Legitimität des verbindlichen Diskurses untergräbt. Das jedoch mit dem Bewusstsein, dass der Satte den Hungrigen nicht versteht und nie verstehen wird. „Das Ohr öffnet sich den Einwänden nur, wenn der Körper den Boden unter den Füßen verliert" - bemerkt in einem ähnlichen Zusammenhang Julia Kristeva (ibidem,1990:27).

Die dritte Perspektive umfasst die Rede des Fremden. Sie sei, behauptet die bulgarisch-französische Autorin, zugleich „barock“ und „nichtig“ (ibidem, 1990:30). Das von dem Fremden in der fremden Sprache ausgesprochene Wort habe keine Chance, sich gegen ,die verbale Leichtigkeit und Eloquenz der angestammten Gesellschaft" durchzusetzen (ibidem, 1990:30). Das, was er sagt, habe in den Augen der Einheimischen keinen Einfluss, keine soziale 
Basis, die letztendlich den Wert der Worte bestimmt. Die Worte des Fremden mögen interessant sein, haben aber „wenig Gewicht gegenüber dem Interesse“ des zuhörenden Gastgebers (ibidem, 1990:30). Diese Worte nützen nichts, spielen keine Rolle im Netz der gesellschaftlichen Beziehungen, haben keine „Stütze in der äußeren Realität“, weil der Fremde ,gerade von dieser abgeschottet bleibt“. „Eure Reden“, sagt Kristeva, „auch wenn sie durch ihre Fremdheit faszinieren, werden keine Folgen, keine Wirkungen zeigen, das Image oder das Ansehen eurer Gesprächspartner nicht heben" (ibidem, 1990:30). Das von Kristeva in diesem Kontext evozierte Bild des „zerstreuten“, manchmal ,amüsierten“ einheimischen Zuhörers, der die Worte des Fremden, seine oft virtuos vorgetragene Rede, nach dem Verklingen des letzten Lautes gleich vergisst, ist ein Schlüsselmotiv in Świderskis Romanen. Der Schweigende und der Redner - in beiden Rollen ist sein Fremder ein Verlierer.

Diese drei Perspektiven, drei Zugänge zu dem Erfahrungsfeld „Migration - Fremdheit - Sprache" erweisen sich bei der Lektüre des Romans Stowa obcego als heuristisch wirksam, denn sie erlauben es dem Leser, tief in die Problematik des Werkes einzudringen.

\section{UND ES WARD ... EIN FREMDER}

Der Roman fängt mit einer Schöpfungsgeschichte der Fremdheit an. Am Anfang war das Wort, und zwar das oppressive Wort des Einheimischen. Der Fremde, den Świderski aus „einem fremden Planeten“ nach Dänemark kommen lässt, hört zunächst Worte, die er nicht versteht (SO, 7). Es sind Worte eines Polizeibeamten, gleichzeitig aber, auf einer symbolischen Ebene, Worte eines Gottes, der ihn mit seinem „es werde..." erschuf - und zwar als einen Fremden. In dem neuen Land bekommt der „Außerirdische“, der Immigrant, ein Ich und wird somit als Fremder (neu-)geboren. Die Worte schaffen den Menschen, indem sie ihn beschreiben (SO, 12). Sie formen seine rational entworfene Identität aus dem Nichts der flüchtigen Emotionen, der unklaren Empfindungen und aus dem Schweigen. Das erste Kapitel des Romans scheint die Schöpfungsgeschichte aus dem Buch Genesis zu parodieren. Der Fremde rekonstruiert seine eigene, private Schöpfungsgeschichte, will das Geheimnis seiner Existenz im Exil durchdringen. Und damit auch das Geheimnis seiner Bestimmung. Denn „obwohl er sich nicht selbst erschaffen hat, muss er doch für seine Worte und Taten Verantwortung tragen" (SO, 13). Worte haben seine Existenz ex nihilo hervorgebracht, aber dies ist durch eine radikale Begrenzung geschehen. Durch die Entnahme der Freiheit, die mit dem Unbestimmten, Offenen zusammenhängt. Am sechsten Tag der Schöpfung ist die ausgrenzende Arbeit der Wortes endlich verrichtet, das Eigene und das entgegengestellte Fremde werden zum oppressiven Grundprinzip der Wirklichkeit (SO, 30). Alle aus diesem Prinzip hervorgehenden 
Begriffe müssen im Rahmen einer entsprechend konstituierten Logik funktionieren, der Gewalt des notwendigen und absoluten Logos untergeordnet. Sich von dieser Gewalt zu befreien, heißt das Wort des einheimischen Gottes rückgängig zu machen und eine neue Schöpfungsgeschichte zu schreiben, die mit einem neuen, eigenen ,es werde..." beginnt. Ist es aber möglich, sich aus der so aufgezwungenen Fremdheit zu befreien? Haben die Worte des im Regime der Fremdheit gefangenen Menschen eine Bedeutung? Diese Fragen leiten über zum zweiten Kapitel des Romans.

\section{DER EINZELNE GEGEN DEN KOLLEKTIVEN DISKURS}

Der Fremde erfährt bald, dass ein Wort in der neuen Wirklichkeit ebenso wenig zählt, wie seine individuelle Existenz. Świderski beschreibt das Immigranten-Dasein in einer Gesellschaft, in welcher das Kollektiv objektiv ist, der Einzelne aber als irrational, sekundär erscheint (SO, 40). Diese Unterschätzung des Einzelnen geschieht paradoxerweise in einem Land, das wohl den größten Apologeten des Einzelnen hervorgebracht hat, nämlich Søren Kierkegaard. Świderski betont dies nachdrücklich, gibt aber oft zu verstehen, dass auch Kierkegaard in der dänischen Gesellschaft als Fremder betrachtet wurde und seine Worte als befremdlich galten. ${ }^{5}$

Die im zweiten Kapitel des Romans geschilderte Vorlesung von Professor Palle Larsen Dantzig zeigt die überwältigende Macht des Wortes eines Einheimischen. Dieses Wort ist deutlich gegen den außenstehenden Einzelnen gerichtet. Es stellt sogar die objektive Existenz des Fremden in Frage. Existiert überhaupt jemand, der als Einzelmensch die Normen der Gesellschaft mit anderen nicht teilt, ihre Tradition nicht kennt, die Sprache der Gesellschaft nicht spricht? - fragt er rhetorisch (SO, 45). Eine solche Existenz erscheint ihm als Anomalie, als Phantomexistenz. Aus der herrschenden Perspektive, präsentiert durch Professor Dantzig, wird der außerhalb des Kollektivs stehende Fremde nicht als Mensch wahrgenommen. Das heißt also, dass eine Selbstbestimmung des Immigranten nicht möglich ist. Er wird als Un-Mensch aus dem Paradies der (dänischen) Gesellschaft vertrieben.

Der Titel des relevanten Kapitels ist „Mowa“, was auf Deutsch „Rede“ und zugleich „Sprache“ heißt - doch im Grunde ist hier der Diskurs gemeint, eine Aussagepraktik, die das Phänomen des „Einheimischen“ konstruiert (vgl. Keller, 2008:75). Es ist der durch Ausgrenzung und Ausschließung sich

${ }^{5}$ In seinen Kierkegaard-Essays unterstrich Świderski mehrmals die in den Texten des dänischen Philosophen zum Ausdruck gebrachte extreme Aufwertung des Individuums, die sich auch in der politischen Sphäre der menschlichen Aktivität zu realisieren habe. In späteren Studien jedoch unterstellt Świderski Kierkegaard, dass er im Kampf gegen den Kollektivismus alle Formen kollektiver Gemeinschaft bekämpfe und sich damit auch gegen die Juden wende (siehe Świderski, 2015:264, 267 und 352-356). 
behauptende Diskurs der Einheimischen, welcher mit der von Kristeva bemerkten „verbalen Leichtigkeit und Eloquenz“ in Professor Dantzigs Rede zum Ausdruck kommt. Es ist eine Eloquenz, die alle Einwände derer, die als Andere, Einzelne auftreten, als nichtig erscheinen lässt. Der Vortrag des Gelehrten zeigt, wie der herrschende Diskurs in der Gesellschaft reproduziert wird: durch das Aktualisieren der „Prozeduren der Ausschließung“, dessen Hauptaufgabe es laut Michel Foucault ist, „die Kräfte und die Gefahren des Diskurses zu bändigen“ (Foucault, 1991:11). Sie dienen also „der Kontrolle des Diskurses von außen“ (Ruoff, 2013:85). Mit dem mächtigen Diskurs konfrontiert schrumpft die individuelle Existenz, sie wird reduziert und devaluiert. „In einem gewissen Grade wurde er schon zu einem Insekt" - lesen wir über den Fremden im letzten Satz des analysierten Kapitels (SO, 46). Die Verwandlung von Gregor Samsa in ein befremdlich und undeutlich sprechendes Ungeziefer liegt hier nicht fern. ${ }^{6}$

\section{5. ÜBERSETZUNG - ZWISCHEN WORT UND DISKURS}

Im dritten Kapitel des Romans („Unpünktlichkeit“) führt Świderski das Motiv einer fremden, unbekannten Sprache ein. „Herr B.“, Dozent am Institut für Slawistik der Universität Kopenhagen mit den Spezialgebieten Polnisch und Übersetzungstheorie, bekommt einige Übersetzungen aus einer asiatischen Sprache zur Begutachtung (SO, 48). Die Auftraggeberin ist seine Bekannte, die Polizeibeamtin Lone, welche angeblich den Fall eines Asylbewerbers untersucht. Sie wird im Roman als eine loyale „Dienerin des Diskurses“ dargestellt. Lone predigt die Ideologie der moralischen Überlegenheit des einheimischen, $d$. h. dänischen Gesellschaftsystems gegenüber anderen, fremden Lebens- und Denkweisen (SO, 48). B. - selbst ein Fremder in Dänemark - soll nun ,mit ihr die möglichen Interpretationen eines Begriffes oder eines Satzes erwägen“ (SO, 49). Als Übersetzungstheoretiker muss er ja wissen „was man tun soll, um aus fremden Zeichen einen verständlichen, dänischen Sinn herauszuholen" (SO, 49). In diesem Zusammenhang erwähnt Lone die von B. entwickelte "Theorie der dritten Sprache“.

Diese Theorie postuliert Übersetzung in eine „dritte“ Sprache, die als „neutral“" erscheint, da sie nicht die Zielsprache, die Sprache des Auftraggebers ist. Weil das „Interesse“ des Originaltextes in der Regel nicht dasselbe ist, wie das „Interesse“ des Zielempfängers, neige - so B. - der Übersetzer dazu, seine Übersetzung in die Richtung des Rezipienten zu „biegen“, d. h. sie

${ }^{6}$ In Kafkas Erzählung Die Verwandlung verunstaltet eine „,von unten her“ kommende unartikulierte nicht-menschliche Stimme die von Samsa ausgesprochenen Worte; sie klingen jetzt anders, fremd (Kafka, 1981:66). Vgl. dazu insbesondere Deleuze \& Guattari (1986: 22-23). 
den Bedürfnissen des Auftragsstellers anzupassen. Und dieses sei im Grunde ein Verrat des Geistes und des Buchstaben des Originals (SO, 50).

Dem Übersetzer liegt also viel daran, dass der Rezipient zufrieden ist. Darum passt er die Übersetzung an die sprachlichen und gesellschaftlichen Normen des Zielrezipienten an (SO, 58). Im Falle eines Immigranten gelten die Normen des Gesetzes (des Grundgesetzes, also der Verfassung) als bestimmend; Lone fasst es kurz zusammen: die für die Polizei angefertigte Übersetzung muss an die Normen des Gesetzes angepasst werden (SO, 58). Die allgemeinste Norm ist die Verfassung des Gastgeberlandes - jede offiziell anerkannte Übersetzung muss sich deshalb nach der Norm der Verfassung richten. Denn das Grundgesetz bestimmt u.a. die landesspezifischen Grenzen des Sprechens, der Kommunikation. Und so geschieht es, dass der Übersetzer meistens vor den Regeln des herrschenden Diskurses kapituliert und das Wort des Fremden aufgibt, opfert.

„Eine Übersetzung, die gleichzeitig dem (unbekannten) Original und dänischen Gesetzen treu sein sollte, stellte eine Herausforderung für B.s Fertigkeiten dar" - stellt der Erzähler fest (SO, 58). In der Tat - und nicht zuletzt, weil hier ein klarer Widerspruch entsteht. Die Theorie der „neutralen“ Übersetzung ist mit einer funktionalen Anpassung nicht vereinbar, denn es ist eine ausdrücklich antifunktionalistische Übersetzungstheorie, die sich gegen die Voraussetzungen der sogenannten Skopos-Theorie richtet, welche die Translation als zweckorientiertes Handeln betrachtet, wobei dieses Zweck immer von Empfänger und Empfangssituation abhängig ist. ${ }^{7}$ Die Befreiung des Übersetzens (und damit auch des Verstehens) von dieser Heteronomie erfolgt durch die Abkehr von Interesse des Auftraggebers. Nur interessenloses Übersetzen vermag das Interesse des Originals so treu wie es nur die Sprachsysteme erlauben wiederzugeben. Sie gibt dem Übersetzer die Chance, das wahre Wort des Fremden vor der Gewalt des herrschenden Diskurses zu retten.

Übersetzung in die dritte Sprache erscheint in Świderskis Roman als Modell eines demokratischen Dialogs, wo das Ziel der Kommunikation das Verstehen des Fremden ist (SO, 191). Es ist zweifelhaft, ob dieses Ziel überhaupt erreichbar ist, aber man sollte zumindest versuchen, sich dem Fremden Original/Individuum durch eine Affirmation seiner Fremdheit anzunähern, da ,erst in der Affirmation der Fremdheit, die in jeder guten Übersetzung zum Ausdruck kommt, sich unser Menschensein vervollständigt" (SO, 195). Der Regime des Eigenen ist immer eine Gefahr für die Übersetzung:

7 „Der Übersetzer oder die Übersetzerin handelt im allgemeinen nicht aus eigenem Antrieb, sondern im Auftrag eines Kunden oder Auftraggebers (...)“, stellt die Funktionalistin Christiane Nord fest (1993:14-15). 
unter seiner Herrschaft muss das Fremde verstummen oder die neue, landeseigene Sprache sprechen.

In Wirklichkeit ist die im Roman thematisierte Übersetzungstheorie eine klare Absage an den Mechanismus der (sozial-politischen) Anpassung und somit auch an den Diskurs der Herrscher. Wenn wir, nach dieser Logik handelnd, das Original nur als „Informationsangebot" für einen konkreten Rezipienten betrachten und auf diese Weise „entthronen“ (Reiß; Vermeer, 1984:100-104), geben wir uns das Recht, originelles, fremdes Wort und originelle, fremde Existenz $\mathrm{zu}$ verändern, an unseren eigenen Diskurs anzupassen. Auf diese Weise verlieren wir das wahre Wort des Fremden, das uns durch seine Unverständlichkeit provoziert und zu einer hermeneutischen Mühe zwingt. Einer Mühe, die uns mit der Einsicht in unsere eigene Fremdheit belohnen kann. Wir sind uns selbst oft fremd und der Andere ist ja im Grunde immer ein Fremder (SO, 182). Die Moral der in Świderskis Roman durchgeführten Analyse liegt offen zutage: dort wo das individuelle Wort von dem allgemeinen Diskurs unterdrückt wird, finden die Menschen nicht zueinander.

Aus dem Blickwinkel des Herrn B. betrachtet, sind die Worte der Fremden auch deshalb interessant und der hermeneutischen Arbeit wert, weil sie unsere heimische Welt aus einer anderen Perspektive beschreiben. Sie gleicht der Perspektive eines uns betrachtenden Außerirdischen, welche in den existenzialistischen Science-Fiction-Romanen von Stanisław Lem oft thematisiert wird (SO, 71). Doch den Vertretern der kommunikationstheoretischen Funktionalismus erscheint die Hermeneutik der individuellen Expression als unsinnig. In der Ordnung des herrschenden Diskurses gleicht diese Expression einem sinnlosen Gestammel, einem Lallen. Es ist die Rede des Barbaros.

Wenn wir dann das von Kristeva analysierte schon funktional übersetzte Wort des Fremdens näher betrachten, erscheint es als ein kraftloses, untergeordnetes und die Unterordnung des Subjektes bestätigendes Wort. Es funktioniert als ein Zeugnis der Ohnmacht des Individuums und der Macht des Diskurses. Dieser bändigt, diszipliniert und unterwirft alles Fremde, um sich vor dem Einfluss des Normwidrigen zu schützen. Denn in der funktionalisierten, kollektivierten Gesellschaft herrsche, so Michel Foucault, „eine tiefe Logophobie“ (Foucault, 1991:33), eine Angst vor dem Unberechenbaren, das den Diskurs infizieren könnte.

Das Original des von Lone übersetzten Textes des Fremden ist interessanterweise in der Erzählung abwesend. B. bekommt den Originaltext nie zu sehen. Das abwesende Wort des Fremden kann als eine stumme Anklage gegen den oppressiven Diskurs gedeutet werden. Die Abwesenheit des Originals symbolisiert die Abwesenheit des Fremden als Menschen. Die Gesellschaftsnormen, der offizielle Diskurs verdrängen den individuellen 
Fremden aus der Sphäre der Kommunikation. Aber auf diese Weise wird auch die Biographie des Einzelnen verdrängt, das Individuelle verliert mit dem Allgemeinen und die Welt wird auf diese Weise ärmer. Die Übersetzung in die dritte Sprache gibt uns die Chance, diese Biographie, die Geschichte des Anderen, kennenzulernen. Deshalb erscheint sie als ,die größte Huldigung, die ein Mensch einem anderen bringen kann - und damit auch sich selbst" (OS, 194).

\section{DIE STRATEGIEN DES WIDERSTANDS}

Der Fremde leistet Widerstand, wehrt sich gegen den herrschenden Diskurs mit dem Schwert der Ironie eines Desillusionierten und dem Schild des Glaubens an ein Jenseits des Diskurses (siehe Kristeva, 1990:20). Diese von Kristeva charakterisierten zwei „Typen der Fremden“ verschmelzen in Świderskis Asystent śmierci zu einer Autor-Figur, die auf die oppressive Wirklichkeit des dänischen Staates mal mit bissiger Ironie, mal mit evangelischen Visionen einer fremdenfreundlichen Gesellschaft reagiert (vgl. Świderski, 2007:348, 349) In Stowa obcego kommt scharfe Ironie selten zum Ausdruck, dafür aber eine utopisch-idealistische Vision der Gesellschaft. In diesem Roman werden zwei Wege des Widerstandes gezeigt. Der eine ist verneinend (via negativa), radikal-anarchistisch, der andere demokratisch, führt aber zu einer inneren Immigration - einem inneren Exil des Schweigens.

„Freunde des Fremden können (...) nur diejenigen sein, die sich selbst gegenüber ein Gefühl der Fremdheit empfinden“, schreibt Kristeva (1990:32). Die Fremdheit - auf verschiedene Weisen empfunden, aber gleichermaßen intensiv erlitten - erscheint als der existenzielle Grund der Kommunikation und Liebesbeziehung zwischen B. und Karina. Sie ist eine Gestalt, welche die These von der intuitiv empfundenen gegenseitigen Nähe der Ausgeschlossenen, der Fremden und Entfremdeten zu versinnbildlichen scheint.

Karina ist eine Außenseiterin, die in einen Teufelskreis von rechtsradikaler Gewalt und Liebe geraten ist. „Die wilde Karina“ ist in Dänemark fremd, denn sie versteht es nicht, sich an die Gesellschaftsnormen anzupassen. Sie hat ein HIV-infiziertes Baby aus einem Krankenhaus entführt - angeblich um es zu retten. (SO, 150, 152) Für Karina existiert Liebe vor jeder rationalen Begründung, jenseits von kollektivem Recht und Unrecht (SO, 152). Ihre Taten können kaum rationalisiert werden, in den Augen der dänischen Gesellschaft sind sie unverständlich und wirken befremdlich. Die Hauptfigur des Romans, B., fühlt sich von Karina angezogen, es entsteht eine emotionale Nähe zwischen den beiden, die aus der Erfahrung einer radikalen Fremdheit erwächst. Macht die unbegreifliche Karina die Existenz des „Einzigen“ (Kierkegaards „hiin Enkelte“; vgl. Świderski, 2007:349) begreifbar? 
Schließlich erscheint B. auch der Selbstmord des Mädchens als eine unbegreifliche Begebenheit, als ein Geheimnis: „Also waren sie einander fremd, obwohl sie ihm so nah war" (SO, 214). Es ist in der Tat die einfachste Formel der Dialektik der Fremdheit. Denn nah waren sie einander als Fremde. Nach Karinas Selbstmord vermutet B., dass sie ,jede Art von Zwang ablehnte“ und „frei sein wollte, um frei wählen zu können“ (SO, 214). Diese negative Freiheit, die auf das unbegrenzte Recht zur Selbstbestimmung, Autonomie pocht, fordert die Wertehierarchie der Normgesellschaft heraus, greift den fremdenfeindlichen Diskurs an, welcher Heteronomie predigt und praktiziert.

Die andere Strategie des Widerstandes wird in dem Kapitel „Zurückhaltung" thematisiert. Dort wird ohne Kommentar ein längerer Brief des anonymen Fremden, dessen Fall die Polizistin Lone untersucht, wiedergegeben. Der Brief ist an die Königin von Dänemark, Margrethe II., gerichtet und kann als eine Impression zum Thema Exil und Sprache betrachtet werden. Der Fremde leidet an einer akuten Sprachlosigkeit. Denn er hat zu der neuen, dänischen Sprache keine innere Beziehung. Der Spracherwerb wird als ein Erwerb von leeren Formen dargestellt, welcher auf eine mechanische, zufällige, unnatürliche Weise verläuft (SO, 236).

Wie ist es möglich - fragt der Fremde - sich selbst mit fremden Worten auszudrücken? Seine dänische Sprachlehrerin gibt ihm eine erstaunliche Antwort: Die Existenz des Immigranten sei „metaphorisch", immer von außen her definiert und bestimmt (SO, 236). Eine Selbstbestimmung ist nur für die Dänen, die Einheimischen reserviert - die Fremden müssen sich mit der Fremdbestimmung abfinden (SO, 237). Der „metaphorische“ Fremde wird so zu einer Figur der Andersheit, des Außerhalbseins. Diese Figur wird den Immigranten von den Einheimischen als eine Art Aufgabe aufgezwungen. Er muss sich von nun an in der vorgegebenen Rolle ,weiterschaffen“, was mit einem Neuanfang verbunden ist. Für den Immigranten fängt eine neue Zeitrechnung an (SO, 239). Die einzig mögliche Gegenstrategie ist in solcher Situation das Schweigen. „Mein Schweigen ist - eben mein und keines anderen“ - schreibt der Fremde in seinem Brief (SO, 236). Es sei ja nicht so, argumentiert er, dass wir ,alles in Worte umbilden müssen, in einen öffentlichen Diskurs“ (SO, 242). Der schweigende Mensch hat de facto keine Rechte, kann aber so seine Freiheit bewahren. Er entflieht der Sprache, die zum Werkzeug des kollektiven Interesses geworden ist, eines Interesses, welches sich auf die Erhaltung des in der Gesellschaft funktionierenden Status quo richtet.

\section{PLÄDOYER FÜR EINE XENOETHIK}

Der Weg des Schweigens führt zu einer neuen Ethik der Fremdheit, welche die Rechte der schweigenden, sprachlosen Minderheit schützt. Der namenlose Fremde richtet sich an die Königin von Dänemark mit der Bitte, die 
Nicht-Einheimischen aus dem Zwang des Diskurses zu befreien und ihre Untertanen zu belehren, dass sie in der Kommunikation mit Fremden eine lautlose Zeichensprache akzeptieren sollten. Es ist tatsächlich eine „dritte Sprache", die uns erlaubt, sich dem anderen Menschen zu nähern. Hier wird der schon erwähnte christlich-utopische Gehalt des Widerstandes sichtbar. Er zieht sich wie ein roter Faden durch Bronisław Świderskis Schaffen und kann als ein ständig modifizierter Entwurf einer Xenoethik aufgefasst werden. In dem Roman Stowa obcego ist dieser Entwurf eng mit der Vorstellung einer Gastfreundschaft verbunden, welche die hierarchische Ordnung des Eigenen und des Fremden dekonstruiert. Sie stimmt weitgehend mit Jacques Derridas Gedanken zur Praxis und Kultur der Gastfreundschaft überein. Eine Kultur der Gastfreudschaft heißt den Fremden im Eigenen willkommen und zeigt einen Respekt für das Andere, für das Idiomatische in ihm, das er in seiner eigenen Sprache äußert. Das Problem der Sprache wird auf diese Weise, wie es Aleksander Kopka treffend bemerkt, zum Hauptproblem der Derridianischen Ethik der Gastfreundschaft (Kopka, 2014:326). Und Derrida selbst fasst es so auf:

Among the serious problems we are dealing with here is that of the foreigner who, inept at speaking the language, always risks being without defense before the law of the country that welcomes or expels him; the foreigner is first of all foreign to the legal language in which the duty of hospitality is formulated, the right to asylum, its limits, norms, policing, etc. He has to ask for hospitality in a language which by definition is not his own, the one imposed on him by the master of the house, the host, the king, the lord, the authorities, the nation, the State, the father, etc. This personage imposes on him translation into their own language, and that's the first act of violence. That is where the question of hospitality begins must we ask the foreigner to understand us, to speak our language, in all the senses of this term, in all its possible extensions, before being able and so as to be able to welcome him into our country? If he was already speaking our language, with all that that implies, if we already shared everything that is shared with a language, would the foreigner still be a foreigner and could we speak of asylum or hospitality in regard to him? (Derrida, 2000a:15)

Und in Bezug auf den Diskurs und den Widerstand des schweigenden Fremden ist diese Bemerkung aus Derridas Step of Hospitality zu lesen:

Inviting, receiving, asylum, lodging, go by way of the language or the address to the other. As Levinas says from another point of view, language is hospitality. Nevertheless, we have come to wonder whether absolute, hyperbolical, unconditional hospitality doesn't consist in suspending language, a particular determinate language, and even the address to the other. Shouldn't we also submit to a sort of holding back of the temptation to ask the other who he is, what her name is, where he comes from, etc.? Shouldn't we abstain from asking another 
these questions, which herald so many required conditions, and thus limits, to a hospitality thereby constrained and thereby confined into a law and a duty? (Derrida, 2000b:133, 135)

Es sind Fragen, die auch Świderskis literarische Auseinandersetzung mit dem Phänomen Fremdheit aufwirft.

Das Praktizieren einer in der Xenoethik verankerten Gastfreundschaft verändert und bereichert den Gastgeber. Es ist eigentlich eine Zumutung des Fremden, die zu einer „Bedingung der Identität“ wird (Maak, 2017). In Świderskis Roman Stowa obcego kehrt dieser Gedanke in vielen Variationen wieder. Auch in der Form einer fundamentalen Frage an die dänische Gesellschaft: Wenn es so ist, dass die Dänen die Worte der Anderen ignorieren, wie können sie sich selbst, ihre eigenen Worte hören? Der Fremde öffnet ja den Weg zum Selbstverstehen, indem er mit seiner Existenz auf das Individuelle, Eigenartige hinweist. ${ }^{8}$ Das Verhältnis zu Fremden sagt viel über die Qualität der zwischenmenschlichen Beziehungen. Der Andere ist ja im Grunde immer ein Fremder (SO, 182). Dort, wo das individuelle Wort von dem allgemeinen Diskurs unterdrückt wird, finden die Menschen nicht zueinander. Solange wir uns nicht von der eigenen Sprache distanzieren, solange wir mit unserer Sprache Oppositionen, Grenzen schaffen, werden wir das Fremde aus unserer Welt ausweisen. Der im letzten Kapitel des Romans aus dem Königreich Dänemark ausgewiesene, zwangsabgeschobene anonyme Fremde erscheint als eine symbolische Gabe - die Gabe den Anderen wahrzunehmen (SO, 253).

\section{LITERATUR}

Deleuze, G., Guattari, F. (1986). Kafka. Toward a Minor Literature. Translated by D. Polan. Minneapolis - London: University of Minnesota Press.

Derrida, J. (2000a). Foreigner Question. In: Dufourmantelle, A., Derrida, J. Of Hospitality (S. 3-73). Translated by R. Bowlby. Stanford: Stanford University Press.

Derrida, J. (2000b). Step of Hospitality / No Hospitality. In: Dufourmantelle, A., Derrida, J. Of Hospitality (S. 75-155). Translated by R. Bowlby. Stanford: Stanford University Press.

Foucault, M. (1991). Die Ordnung des Diskurses. Aus dem Franz. von W. Seitter. Frankfurt am Main: Fischer.

Kafka, F. (1981). Die Verwandlung. In: F. Kafka, Sämtliche Erzählungen. Hrsg. von P. Raabe (S. 64-113). Frankfurt a.M. und Hamburg: S. Fisher.

Keller, R. (2008). Michel Foucault. Konstanz: UVK.

Kopka, A. (2014). Pytanie o gościnność w filozofii Jacques'a Derridy. Folia Philosophica 32, 319-335.

Kristeva, J. (1990). Fremde sind wir uns selbst. Aus dem Franz. von X. Rajewsky. Frankfurt am Main: Suhrkamp.

${ }^{8}$ Vgl. hier die Analogie mit „Kierkegaards Methode“(Świderski, 2007:358). 
Maak, N. (2017). Der Fremde schafft uns ein Zuhause. Frankfurter Allgemeine Zeitung. 1.8.2017. http://www.faz.net/aktuell/feuilleton/buecher/buch-von-der-gastfreundschaft-der-fremdeschafft-uns-ein-zuhause-15129324.html (25.2.2018).

Nord, Ch. (1993). Einführung in das funktionale Übersetzen. Am Beispiel von Titeln und Überschriften. Tübingen und Basel: Francke.

Reiß, K., Vermeer, H.J. (1984). Grundlegung einer allgemeinen Translationstheorie. Tübingen: Max Niemeyer.

Ruoff, M. (2013). Foucault-Lexikon. Entwicklung - Kernbegriffe - Zusammenhänge. 3. Aufl. Paderborn: Wilhelm Fink.

Słowa obcego, Bronisław Świderski. https://www.znak.com.pl/kartoteka,ksiazka,1997,Slowaobcego.html (15.2.2018).

Świderski, B. (1998). Stowa obcego. Kraków: Znak.

Świderski, B. (2007). Asystent śmierci. Powieść o karykaturach Mahometa, o mitości i nienawiści w Europie. Warszawa: WAB.

Świderski, B. (2012a). Doświadczenia emigranta. In: B. Świderski, Kiedy mogę zabić? Dyskusje o kulturze i przemocy. Red. J. Borowczyk, M. Larek (S. 111-113). Poznań: Wydawnictwo WBPiCAK.

Świderski, B. (2012b). Znowu do diabła ten etos! In: B. Świderski, Kiedy mogę zabić? Dyskusje o kulturze i przemocy. Red. J. Borowczyk, M. Larek (S. 118-136). Poznań: Wydawnictwo WBPiCAK.

Świderski, B. (2015). Czepiam się Kierkegaarda. Warszawa: Fundacja Augusta Hrabiego Cieszkowskiego.

\section{Piotr de Bończa Bukowski}

Instytut Filologii Germańskiej

Zakład Filologii Szwedzkiej

Uniwersytet Jagielloński w Krakowie

Al. Mickiewicza 9A

31-120 Kraków

Polska

piotr.bukowski@uj.edu.pl 